EL EFECTO DE LA EPOCA DE SIEMBRA, EL GENOTIPO Y LA FERTILIDAD SOBRE EL RENDIMIENTO EN GRANO DE TRIGO CANDEAL EN LA REGIÓN SUBHÚMEDA-SECA PAMPEANA

\title{
THE EFFECT OF THE SOWING DATE, GENOTYPE AND THE FERTILITY ON GRAIN YIELD OF DURUM WHEAT IN THE DRY-SUBHUMID PAMPAS REGION
}

Fernández, Miguel Angel ${ }^{1 *}$, Gaggioli, Carolina ${ }^{1}, y$ Repollo Rodolfo ${ }^{1}$

\section{RESUMEN}

Los cereales invernales son importantes en la región sub-húmeda seca pampeana. En la provincia de La Pampa no se cultiva trigo candeal, no obstante, éste se adapta a zonas semiáridas del mundo. El objetivo fue analizar herramientas de manejo que permitan su incorporación al agrosistema pampeano, tales como: el genotipo, la época de siembra y la fertilidad. Se utilizaron cuatro genotipos de trigo candeal, en dos épocas de siembra y en dos condiciones de fertilidad. Los ensayos fueron realizados en el Campo Experimental de la Facultad de Agronomía de la UNLPam., ubicado en $36^{\circ}$ 32 ' 49" S y $64^{\circ} 18^{\prime} 20^{\prime \prime} \mathrm{W}$. La primera época de siembra mostró mayor rendimiento que la segunda, pero se detectaron interacciones del genotipo con la época. El agregado de fertilizante nitrogenado aumentó el rendimiento en las dos fechas de siembra. Existe variabilidad genética, en la siembra más temprana el mayor rendimiento en grano lo tuvo Buck zafiro en las dos condiciones de fertilidad; y en la tardía, Buck esmeralda con buena fertilidad nitrogenada y Bon. INTA Cariló cuando hay escasez de nitrógeno. Buck esmeralda mostró buena estabilidad de rendimiento en grano en las dos épocas con condiciones adecuadas de fertilidad.

PALABRAS CLAVE: trigo fideos; fecha de siembra; variedades; nitrógeno

\section{ABStRAct}

Winter cereals are important in the subhumid dry pampas region. In La Pampa province is not cultivated durum wheat, however, it is adapted to semiarid zones of the world. The objective was to analyse management tools that allow their incorporation to pampean agrosystem, such as: genotype, sowing time and fertility. Four genotypes of durum wheat were used, in two sowing times and in two fertility conditions. The trials were conducted in the Experimental field of the Faculty of Agronomy of the UNLPam, located in $36^{\circ} 32^{\prime} 49^{\prime \prime S}$ and $64^{\circ} 18^{\prime} 20^{\prime \prime} \mathrm{W}$. The first sowing date showed greater yield than the second, but genotype interactions were detected with the time. The addition of nitrogen fertilizer increased yield in the two sowing seasons. There is genetic variability, in the earliest sowing the highest yield grain had Buck zafiro in both conditions of fertility; and in the late, Buck esmeralda with good nitrogen fertility, and Bon. INTA cariló when there is a shortage of nitrogen. Buck esmeralda showed good grain yield stability in the two times with good fertility conditions.

KEY WORDS: Wheat noodles; sowing date; varieties; nitrogen

\section{INTRODUCCIÓN}

Los cereales de invierno son importantes en la producción agropecuaria de la región subhúmeda seca pampeana (Pascale \& Damario,

Cómo citar este trabajo:

Fernández, M. A., Gaggioli, C., y Repollo, R.. (2019). El efecto de la epoca de siembra, el genotipo y la fertilidad sobre el rendimiento en grano de trigo candeal en la región subhúmeda-seca pampeana. Semiárida, 29(2), 51-60.
2004). En esta región el cereal de invierno más difundido es el trigo pan (Triticum aestivum L.). En La Pampa, no se registra actualmente el cultivo de trigo candeal (Triticum durum Desf.), si bien entre los años 2000 y 2009 se registraron unas 300 ha (MAGyP, 2018). No obstante, el trigo candeal es cultivado en zonas semiáridas y de alta variabilidad climática del mundo, que reciben menos de $350 \mathrm{~mm}$ durante

1 Universidad Nacional de La Pampa, Facultad de Agronomía, La Pampa, Argentina

*mfernandez@agro.unlpam.edu.ar 
Fernández, M. A., Gaggioli, C., y Repollo, R.

el ciclo y es considerado más tolerante a la sequía que el trigo pan (Bozzini, 1988). La diversificación de los cultivos aumenta la estabilidad y reduce el riesgo de los sistemas agropecuarios (Loomis \& Connor, 2002; Tanaka et al., 2002). Si bien existen antecedentes de su cultivo en la región se conoce poco de su adaptación.

Los factores determinantes y limitantes en la definición del rendimiento en grano que permiten la adaptación a una región son fuertemente modificados por la elección de la fecha de siembra y define la exploración del cultivo en cuanto a las condiciones de radiación, temperatura y precipitaciones, en el período crítico. Además, a diferencia de otras prácticas agronómicas no implica un aumento significativo de los costos de producción (Otegui \& López Pereira, 2003).

La disminución del rendimiento en trigo candeal a medida que se atrasa la fecha de siembra fue reportada por numerosos autores (Anwar et al., 2007; Forster et al., 2017; Patel et al., 2018). Sin embargo, en varios ambientes del sudeste de la región pampeana (Arg.), en ensayos comparativos de rendimiento en grano con épocas de siembra, la última (principio de agosto) tuvo más rendimiento (Larsen \& Jensen, 2016).

El rendimiento está fuertemente asociado al número de granos por unidad de superficie, aunque cuando se fijan pocos, normalmente, se obtienen granos con un mayor peso individual y el ambiente tiene gran influencia sobre ambos (Evans \& Wardlaw, 1976). Debido a que el número de granos por metro cuaddrado se determina en un momento del ciclo y el peso de los granos en otro, se puede asociar la variación del rendimiento y de los componentes que se generan en cada período a las limitantes ambientales y bióticas que sucedan en cada uno de ellos (BrancourtHulmel et al., 1999).

El trigo candeal produjo menos número de granos que el trigo pan, principalmente por una menor fertilidad de las espiguillas (Abbate et al., 1998). En ambientes con estrés se ha propuesto que en trigo candeal el aporte del peso de los granos al rendimiento se torna significativo (González-Ribot et al., 2017).

En una zona bajo riego (Villarino, Bs. As.), la fertilización nitrogenada incrementó el rendimiento en grano del cultivo, maximizándose a la dosis de $100 \mathrm{~kg}$ de N.ha ${ }^{-1}$ aplicada en forma fraccionada $(50 \%$ a la siembra y 50\% al macollaje) (Cantamutto et al., 1994).

Los diferentes genotipos presentaron adaptación a distintos lugares y épocas de siembra en un estudio realizado en la zona triguera IV (Larsen \& Jensen, 2016).

La fecha de siembra y la aplicación de fertilizantes pueden modificar el desarrollo del cultivo, expresado en el número de granos potenciales por unidad de superficie para su posterior llenado (Dennett, 1999).

\section{Hipótesis:}

a) La producción de granos del trigo candeal disminuirá con el atraso en la época de siembra,

b) La fertilización nitrogenada aumentará el rendimiento en grano dentro de cada fecha de siembra.

c) Existirá variabilidad genética que aporte adaptación a la región.

Objetivos:

Evaluar el rendimiento de grano $\left(\mathrm{kg} \cdot \mathrm{ha}^{-1}\right) \mathrm{y}$ los componentes del mismo y su variación por el efecto de la época de siembra, el genotipo y la fertilidad.

\section{Materiales y Métodos \\ Área de estudio}

Los ensayos fueron realizados en el año 2017 en el Campo Experimental de la Facultad de Agronomía de la UNLPam., ubicado en $36^{\circ} 32^{\prime}$ $49^{\prime}$ S y $64^{\circ} 18^{\prime} 20^{\prime \prime} \mathrm{W}$, a $210 \mathrm{msnm}$. La región se ha clasificado como subhúmeda-seca para cultivos invernales, en base a la humedad disponible en el período crítico que es el mes alrededor de la espigazón (entre - 1 y -25 mm de deficiencia hídrica) (Pascale \& Damario, 2004). 
El efecto de la epoca de siembra, el genotipo y la fertilidad sobre el rendimiento en grano de trigo candeal en la región subhúmedaseca pampeana

El suelo de dicho lote se ha re-clasificado recientemente como Paleustol petrocálcico, debido a cambios generados por el Soil Survey Staff (2014), con las proporciones de arcilla, limo y arena, 10, 20 y $70 \%$, respectivamente, con escasa pendiente superficial y un manto de tosca en el subsuelo, a una profundidad que varió entre $0,8 \mathrm{~m}$ y $1,0 \mathrm{~m}$. Los parámetros del suelo evaluados al momento de la siembra fueron: materia orgánica; $1,17 \%$; fósforo disponible (Bray I); 17,6 ppm y nitrógeno de nitratos: 6,8 y 5,0 ppm a $0-30$ y $30-60 \mathrm{~cm}$ de profundidad respectivamente.

\section{Material vegetal}

Los genotipos fueron elegidos de acuerdo a la disponibilidad en el mercado zonal: Bonaerense INTA Facón (año de inscripción en CONASE, 1998), Buck esmeralda (año de inscripción en CONASE, 2000), Bonaerense INTA Cariló (año de inscripción en CONASE, 2004) y Buck Zafiro (año de inscripción en CONASE, 2015).

Las fechas de siembra fueron: 14 de julio de 2017 y 3 de agosto de 2017. Se descartó estudiar una fecha más temprana debido al peligro de heladas tardías en el momento de floración. Las densidades de siembra de la primera y la segunda época fueron de 280 y 350 semillas viables por metro cuadrado, respectivamente. $\mathrm{La}$ siembra fue realizada sobre una cama de siembra convencional con barbecho previo en base a labores mecánicas de repaso. El cultivo antecesor en los tres años fue una pastura asociada entre alfalfa (Medicago sativa L.) y festuca (Festuca arundinacea Schreb.).

\section{Diseño experimental}

El diseño experimental utilizado fue en bloques completamente aleatorizados, con tres réplicas de cada tratamiento en macro-parcelas de 10 metros de largo por 3 metros de ancho; con 15 surcos separados a 0,20 metros entre ellos. La fertilidad del suelo fue modificada con un "arrancador" en todas las parcelas mediante el agregado de $80 \mathrm{~kg} \cdot \mathrm{ha}^{-1}$ de fosfato diamónico a la siembra. Al tratamiento denominado "fertilizado" se le agregó $100 \mathrm{~kg} \cdot \mathrm{ha}^{-1}$ de urea al voleo en macollaje temprano (Z15-21 de Zadoks et al., 1974). El control de las malezas fue realizado con herbicidas en el estado Z14 de
Zadoks et al. (1974). Se utilizó una combinación de Metsulfurón Metil (6 g i.a.ha $\left.{ }^{-1}\right)$ y Dicamba (100 cc.ha-1).

\section{Mediciones en el cultivo}

Estados fenológicos. para observar la ocurrencia estados fenológicos más importantes se utilizó la clave de estados de desarrollo descriptos por Zadoks et al. (1974): a) Estado 10, emergencia de las plántulas, b) Estado 65, emergencia de anteras fuera de la espiga, y c) Estado 90, madurez fisiológica, detectado visualmente por el amarillamiento total de la espiga.

Componentes de Rendimiento. Los componentes se valoraron sobre una superficie de un metro cuadrado, tal como lo recomiendan Bell \& Fischer (1994). a) Número de espigas: se realizó el recuento total de espigas fértiles a cosecha. b) Número de granos por espiga: se tomaron 10 espigas al azar y se las trilló manualmente para determinar el componente. c) Peso de los granos: se tomaron al azar dos muestras de 200 granos por parcela y fueron llevadas a peso constante en estufa a $60^{\circ} \mathrm{C}$ durante 48 horas, para calcular el PMG. d) Rendimiento de grano: Se determinó trillando un metro cuadrado del cultivo.

Las muestras de grano obtenidas fueron llevadas a peso constante en estufa a $60^{\circ} \mathrm{C}$ durante 48 horas. El rendimiento se expresó en kg.ha ${ }^{-1}$ a una humedad del grano de $14 \% \mathrm{BH}$.

Otras determinaciones. a) Biomasa aérea: Se cortó a nivel el suelo un metro cuadrado del cultivo que fue pesado luego de ser secados en estufa a $60^{\circ} \mathrm{C}$ hasta peso constante. b) Índice de Cosecha (IC): el cociente entre el rendimiento de grano la biomasa aérea, ambos tomados con el peso a salida de la estufa.

\section{Análisis estadístico}

El análisis estadístico de cada experimento (época) se realizó por medio de Análisis de la Varianza (ANAVA) para evaluar el efecto de los tratamientos sobre cada variable (Steel \& Torrie, 1989). Se utilizó la prueba de Tukey para determinar la diferencia mínima significativa que permitió la separación de las medias de los tratamientos. 
Fernández, M. A., Gaggioli, C., y Repollo, R.

El análisis conjunto de los años se realizó como grupo de experimentos (Steel \& Torrie, 1989). Primeramente se compararon las varianzas (cuadrado medio del error, CME) y así se determinó la posible heterogeneidad de las mismas. Se utilizó el criterio de Box citado por Pimentel Gomes (1978) quien mencionó que el cociente entre el CME mayor y el CME menor no debe ser superior a tres.

\section{Resultados y Discusión \\ Información climática}

En la Tabla 1 se describe el año desde el punto de vista climático (temperatura y lluvias). Las precipitaciones mensuales del año 2017 fueron buenas para el crecimiento del trigo, solamente fueron menores al promedio en el mes de octubre justamente cuando ocurre el periodo crítico. Las temperaturas medias mensuales fueron un poco más altas al promedio en julio y agosto, y luego en la primavera, algo más frescas que el promedio.

\section{Fenología}

La duración del ciclo total de crecimiento de los cuatro genotipos fue semejante dentro de cada época (Z10 a Z90 de Zadoks et al., 1974) (Figura 1). El período desde la emergencia hasta la floración (Z10 a Z65) fue similar con la particularidad que Buck esmeralda y Bon. INTA Facón fueron ligeramente más cortas que las otras dos. El período de llenado de los granos (desde Z65 al Z90) de la segunda época se acortó entre cuatro y cinco días con respecto a la primera. La época de siembra tardía aceleró principalmente el período que va desde Z10 a Z65, acortándose entre 14 y 16 días.

\section{Rendimiento}

Para la evaluación del efecto de la época de siembra sobre el rendimiento en grano debió compararse primeramente el cuadrado medio del error (CME) de los ensayos de cada época. La primera época mostró un CME experimental de 270618, mientras que la segunda de 121704 . Con el objetivo de determinar la homogeneidad de las varianzas de los ensayos y así poder realizar el análisis conjunto como grupo de experimentos se realizó el cociente entre ellos arrojando un valor de 2,2, aceptándose entonces de acuerdo al criterio de Box citado por Pimentel Gomes (1978) que las varianzas son semejantes.

El análisis del rendimiento en grano de trigo candeal en las dos épocas como grupo de experimentos permitió diferenciarlas estadísticamente (Tabla 2). La primera época tuvo mayor rendimiento que la segunda, pero se detectaron interacciones del genotipo con la época de siembra y con la condición de fertilidad, solamente no se detectó la interacción triple. Las interacciones observadas obligaron a estudiar el efecto del genotipo y de la fertilidad de cada época de siembra por separado.

El rendimiento en grano de los cuatro genotipos disminuyó con el atraso en la época de siembra, aunque esta disminución fue de distinta magnitud de acuerdo al genotipo de que se trate, lo que generó la interacción (Tabla 3 ). Semejantes resultados obtuvieron Patel et al., (2018) en Gujarat (India). También hubo distinto comportamiento de la época y el genotipo con el agregado de fertilizante nitrogenado, pero en general aumentó con la fertilización. En la primer fecha, Buck zafiro presentó mayor rendimiento que los demás cultivares, si bien las diferencias no fueron significativas en el tratamiento fertilizado. En tanto que en la segunda fecha, Buck esmeralda estuvo entre los más rendidores cuando se fertilizó pero su comportamiento empeoró en el tratamiento testigo, en relación a los demás genotipos.

Tabla 1. Precipitación mensual y temperatura media mensual de Santa Rosa (LP)

Table 1. Monthly precipitation, and monthly average temperature of Santa Rosa (LP)

\begin{tabular}{lrrrrrrrrrrrrr}
\hline & Ene & Feb & Mar & Abr & May & Jun & Jul & Ago & Sep & Oct & Nov & Dic & AÑO \\
\hline Precip. 2017 (mm) & 71,7 & $93,3320,7$ & 165,7 & 103,7 & 69,3 & 27,9 & 25,9 & 92,9 & 34,9 & 88,3 & 56,5 & 1150 \\
Precip. Media (1975-2017) $(\mathrm{mm})$ & 87,0 & 84,8 & 96,2 & 64,6 & 33,5 & 16,4 & 19,6 & 26,1 & 46,3 & 81,3 & 84,8 & 95,7 & 740 \\
Temperatura media 2017 $\left({ }^{\circ} \mathrm{C}\right)$ & 24,8 & 23,7 & 19,9 & 15,3 & 11,9 & 9,2 & 9,8 & 10,7 & 12,3 & 15,0 & 17,7 & 22,4 & 16,1 \\
To Media Histórica $(1977-2017)\left({ }^{\circ} \mathrm{C}\right)$ & 23,4 & 22,2 & 19,7 & 15,4 & 11,5 & 8,3 & 7,8 & 9,9 & 12,4 & 15,8 & 19,2 & 22,2 & 15,6 \\
\hline
\end{tabular}

Fuente: Datos suministrados por la Cátedra Agrometeorología Facultad de Agronomía UNLPam 
El efecto de la epoca de siembra, el genotipo y la fertilidad sobre el rendimiento en grano de trigo candeal en la región subhúmedaseca pampeana

Tabla 2. ANAVA conjunto de dos épocas de siembra, el genotipo y la fertilidad sobre el rendimiento en grano $\left(\mathrm{kg} \mathrm{ha}^{-1}\right)$

Table 2. ANOVA joint of two sowing date, genotype and fertility on the grain yield $\left(\mathrm{kg} \cdot \mathrm{ha}^{-1}\right)$

\begin{tabular}{lrrrrr}
\hline $\begin{array}{l}\text { Fuente de } \\
\text { Variación }\end{array}$ & \multicolumn{1}{c}{ SC } & gl & \multicolumn{2}{l}{ CM } & \multicolumn{2}{l}{ F } \\
\hline Bloque & 4876845 & 2 & 2438423 & 12,43 & $<0,0001$ \\
Época & 8180561 & 1 & 8180561 & 20,45 & 0,0456 \\
Epoca*Bloque & 800224 & 2 & 400112 & 2,04 & 0,1371 \\
Genotipo & 831518 & 3 & 277173 & 1,41 & 0,2455 \\
Fertilidad & 24515728 & 1 & 24515728 & $124,98<0,0001$ \\
Ép. x Gen. & 2509939 & 3 & 836646 & 4,27 & 0,0077 \\
Ép. x Fert. & 1010784 & 1 & 1010784 & 5,15 & 0,026 \\
Gen. x Fert. & 2795177 & 3 & 931725 & 4,75 & 0,0043 \\
Ép. x Gen. x Fert. & 841672 & 3 & 280557 & 1,43 & 0,2405 \\
\hline Error & 14908273 & 76 & 196161 & & \\
CV (\%) & 16,93 & & & & \\
\hline
\end{tabular}

Obs: $\mathrm{Ep} .=$ época, $\mathrm{Gen}=$ genotipo, Fert. $=$ fertilidad, $\mathrm{CM}=$ cuadrado medio, $\mathrm{F} .=$ Cociente entre los $\mathrm{CM}$ y $\mathrm{P}=$ probabilidad

Destacándose entonces su respuesta al agregado de N (Tabla 3).

No se pudo detectar una asociación entre el rendimiento en grano de los genotipos y el año de inscripción en la CONASE, aunque la variedad más nueva Buck zafiro fue la de mayor rendimiento en grano promedio.

\section{Biomasa aérea y Partición}

La producción de biomasa aérea presentó en general una tendencia similar al rendimiento. Resultó mayor en la primera época de siembra, comportamiento parecido al encontrado por Patel et al., (2018). Además, en la primera época hubo diferencias significativas entre genotipos (Buck zafiro mostró la mayor biomasa), mientras que en la segunda no se detectaron diferencias entre genotipos (Tabla 3). El fertilizante nitrogenado en las dos épocas produjo un aumento de la biomasa. El IC se redujo con la época de siembra sólo en el genotipo Buck zafiro. En los otros tres genotipos el fertilizante nitrogenado aumentó el IC excepto en INTA Cariló en la segunda fecha de siembra.

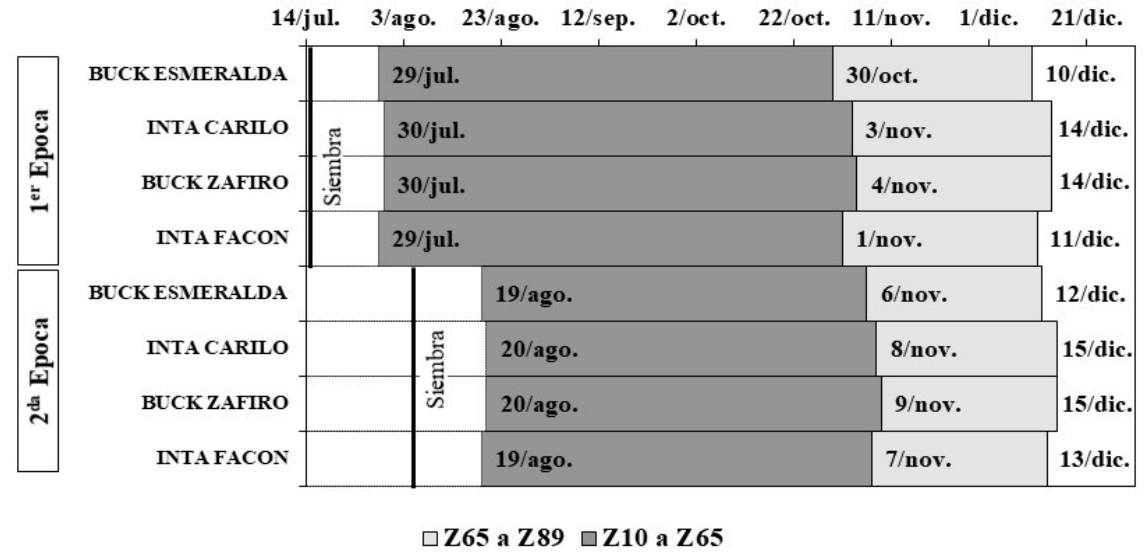

Figura 1. Efecto de la época de siembra sobre el desarrollo de las tres especies, en tres años. Referencias: Z10: emergencia del cultivo; Z65: antesis plena y Z89: fin del llenado grano. Los estados fenológicos fueron determinados de acuerdo al código descrito por Zadoks et al. (1974)

Figure 1. Effect of sowing date on the stages of development of the durum wheat genotypes. References: Z10: crop emergence; Z65: full anthesis and Z89: end of grain filling. Phenological states were determined according to the code described by Zadoks et al. (1974) 
Fernández, M. A., Gaggioli, C., y Repollo, R.

Tabla 3. Efecto de la época de siembra, el genotipo y la fertilidad sobre el rendimiento en grano, la biomasa aérea y el índice de cosecha

Table 3. Effect of sowing date, genotype and fertility on grain yield, the aboveground biomass and harvest index

\begin{tabular}{|c|c|c|c|c|c|c|c|c|c|c|c|c|}
\hline & \multicolumn{4}{|c|}{ Rendimiento en grano $\left(\mathrm{kg} \cdot \mathrm{ha}^{-1}\right)$} & \multicolumn{4}{|c|}{ Biomasa aérea $\left(\mathrm{kg} \mathrm{ha}^{-1}\right)$} & \multicolumn{4}{|c|}{ IC } \\
\hline & \multicolumn{2}{|c|}{ Primera } & \multicolumn{2}{|c|}{ Segunda } & \multicolumn{2}{|c|}{ Primera } & \multicolumn{2}{|c|}{ Segunda } & \multicolumn{2}{|c|}{ Primera } & \multicolumn{2}{|c|}{ Segunda } \\
\hline & $\mathrm{F}$ & $\mathrm{T}$ & $\mathrm{F}$ & $\mathrm{T}$ & $\mathrm{F}$ & $\mathrm{T}$ & $\mathrm{F}$ & $\mathrm{T}$ & $\mathrm{F}$ & $\mathrm{T}$ & $\mathrm{F}$ & $\mathrm{T}$ \\
\hline Zafiro & 3789 a & $2892 b$ & $2412 b$ & $1990 a$ & $10747 a$ & $8344 b$ & $7857 \mathrm{ab}$ & 6753 & 3 bc $0,35 a$ & $0,35 a$ & $0,31 \mathrm{c}$ & $0,30 \mathrm{~b}$ \\
\hline Facón & 3612 a & 1924 c & 2748ab & 1873ab & $9902 a$ & $6948 c$ & 7955a & 5779 & c $0,36 a$ & $0,28 c$ & $0,34 a b$ & $0,32 \mathrm{a}$ \\
\hline Esmeralda & $3431 \mathrm{ab}$ & $2116 c$ & $3192 a b$ & 1676 b & $9675 a$ & 7345 bc & c 8831a & 5649 & c $0,36 a$ & $0,28 c$ & $0,36 \mathrm{ab}$ & $0,30 b$ \\
\hline Cariló & $3229 a b$ & 2265 c & $2553 b$ & $2143 a$ & $9805 a$ & $7597 \mathrm{bc}$ & c 7987a & 6623 & с $0,33 a$ & $0,30 b$ & $0,32 \mathrm{bc}$ & $0,32 a$ \\
\hline Int. $G \times F$ & \multicolumn{2}{|c|}{ NS } & \multicolumn{2}{|c|}{${ }^{*} \mathrm{P}=0,001$} & \multicolumn{2}{|c|}{ NS } & \multicolumn{2}{|c|}{ NS } & \multicolumn{2}{|c|}{${ }^{*} P=0,0006$} & \multicolumn{2}{|c|}{${ }^{*} \mathrm{P}=0,0002$} \\
\hline DMS & \multicolumn{2}{|c|}{608} & 532 & 300 & \multicolumn{2}{|c|}{1244} & \multicolumn{2}{|c|}{1132} & 0,036 & 0,024 & 0,024 & 0,014 \\
\hline CV (\%) & \multicolumn{2}{|c|}{17,9} & 16,1 & 12,9 & \multicolumn{2}{|c|}{12,1} & \multicolumn{2}{|c|}{13,5} & 9,8 & 4,4 & 3,8 & 6,3 \\
\hline
\end{tabular}

Referencias: Primera y segunda = épocas de siembra. $\mathrm{F}=$ fertilizado; $\mathrm{T}=$ testigo. Int. $\mathrm{G}$ x $\mathrm{F}=$ interacción genotipo por fertilidad. NS = no significativo. Letras iguales no difieren estadísticamente con la prueba de LSD $(\mathrm{P} \leq 0,05)$ dentro de la época en el caso de no detectarse interacción y dentro de una columna en el caso de existir interacción

References: First and second = planting times. $\mathrm{F}=$ fertilized; $\mathrm{T}=$ control. Int. $\mathrm{G} \times \mathrm{F}=$ fertility genotype interaction. $\mathrm{NS}=$ not significant. Equal letters do not differ statistically with the LSD test $(\mathrm{P} \leq 0.05)$ within the planting time in case no interaction is detected and within a column in the case of interaction

\section{Componentes del rendimiento}

A medida que el ciclo ontogénico del cultivo avanza quedan definidos los distintos componentes numéricos del rendimiento de grano. En general, estos se pueden englobar en dos macro-componentes principales: la cantidad de granos por metro cuadrado $\left(\mathrm{N}^{\circ}\right.$ gr.m $\left.{ }^{-2}\right)$ y el peso de los mismos. El primero es un componente primordial en la determinación del rendimiento de la mayoría de los cultivos anuales de cosecha y en el cultivo de trigo queda definido en el momento del cuaje de los frutos; unos días después de la antesis (Fischer, 1985).

La cantidad de granos fue mayor en la primera época que en la segunda en los genotipos del criadero Buck (zafiro y esmeralda) y fue poco afectado por la época de siembra en los genotipos del criadero de INTA (cariló y facón). El fertilizante nitrogenado en las dos épocas produjo un aumento de este componente, principalmente por el aumento en la cantidad de espigas (Tabla 4). En la primera fecha, Buck zafiro produjo mayor cantidad de granos que el resto de los genotipos, mientras que en la segunda fecha no hubo diferencias significativas.

La cantidad de espigas $\left(\mathrm{N}^{\circ}\right.$ esp. $\left.\mathrm{m}^{-2}\right)$ aumentó con la nutrición nitrogenada en 50 espigas mas en promedio de los cuatro genotipos en las dos épocas, aunque dentro de los genotipos, Buck esmeralda fue el de menor respuesta (Tabla 4). El atraso de la fecha de siembra no disminuyó marcadamente la cantidad de espigas de acuerdo con lo observado por Schwarte et al. (2006) y contrapuesto a lo reportado por Green et al. (1985) y Spaner et al. (2000) quienes detectaron una disminución de este componente con el retraso de la época de siembra. La cantidad de espigas que se obtuvo en las dos épocas es semejante comparado al estudio realizado en Gujarat, India (Patel et al., 2018).

La cantidad de granos por espiga $\left(\mathrm{N}^{\circ}\right.$ gr.esp $\left.{ }^{-1}\right)$ no fue modificada por la época de siembra (Tabla 4). Resultados semejantes fueron obtenidos por Arduini et al., (2018) en tres épocas de siembra realizadas en Pisa (Italia), mientras que en un sitio más cálido (Gujarat, India) se observó una reducción de este componente al atrasar la fecha de siembra (Patel et al., 2018). La fertilización nitrogenada mejoró la fecundación y sostener en el cuaje alrededor de cinco granos más por espiga que el testigo.

El peso de los granos decayó en tres genotipos (exceptuando a Buck esmeralda), con el retraso de la época de siembra, lo que pudo ser causado 
El efecto de la epoca de siembra, el genotipo y la fertilidad sobre el rendimiento en grano de trigo candeal en la región subhúmedaseca pampeana

por el acortamiento en 4 o 5 días de la fase de llenado de granos.

Otro autores no observaron una caída del peso de los granos al atrasar la fecha de siembra (Arduini et al., 2018; Forster et al., 2017). En cuanto a los cultivares, se observó un elevado PMG de Bon. INTA Facón en la primera época y de Buck esmeralda en la segunda. En tanto que Bon. INTA cariló y Buck zafiro presentaron en general menor peso de granos.

El fertilizante nitrogenado en las dos épocas produjo una pequeña reducción del peso de los granos, esto podría explicarse por una mayor fecundación en posiciones distales de la espiga que generalmente son granos de menor tamaño (Pei et al., 2008; Ferrante et al., 2015).

En general, la reducción de rendimiento en grano (promedio de todos los genotipos) de la segunda época con respecto a la primera fue de $22,5 \%$ y $16,5 \%$ en los tratamientos fertilizados y testigos, respectivamente. Los macrocomponentes del rendimiento aportaron a esta reducción de la siguiente forma: en los tratamientos fertilizados se redujo en una mayor

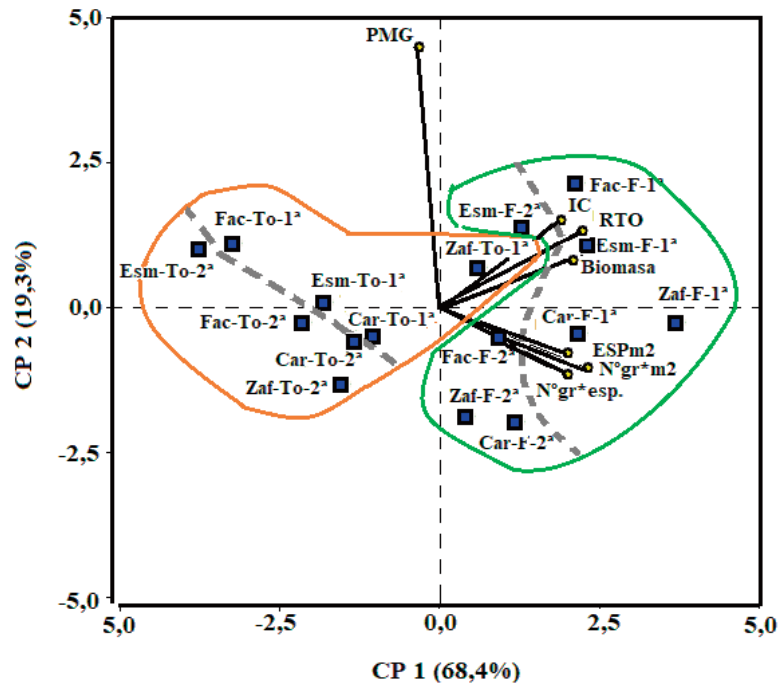

Figura 2. Biplot de análisis de componentes principales (PCA) del efecto del genotipo, la fecha de siembra y la fertilidad sobre los componentes y el rendimiento en grano de trigo candeal

Figure 2. Biplot for principal components analysis (PCA) of the effect of genotype, sowing date and fertility on the components and the grain yield of durum wheat 


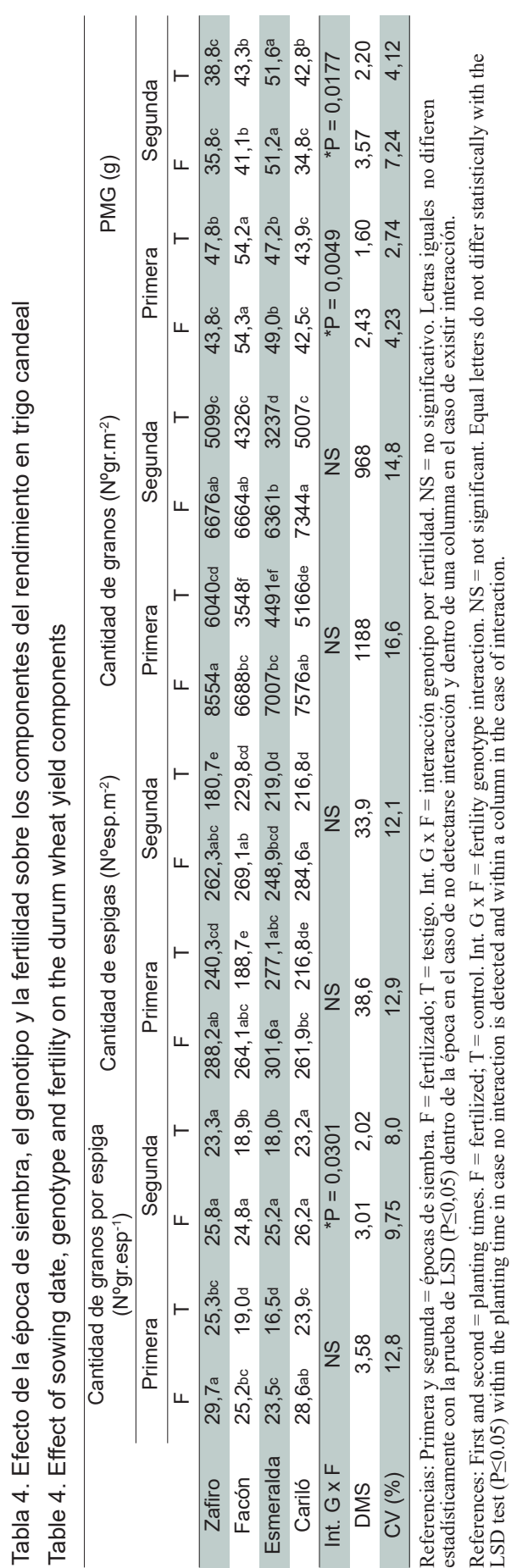

logró explicar 87,7\%. En este biplot se observan dos grandes grupos que están encerrados con línea llena; el de la izquierda engloba a todos los tratamientos testigos (sin aplicación de urea a macollaje) y opuesto al cuadrante donde se ubica la variable rendimiento indicando que éstos fueron menores. El grupo de la derecha involucra a los tratamientos fertilizados y están ubicados cercanos a la variable rendimiento indicando que éstos fueron mayores. A su vez, dentro de cada gran grupo se dividió con línea punteada en dos sectores; el de la izquierda la segunda época de siembra y el de la derecha la primera época de siembra que en general estuvo asociada a mayor rendimiento. De los componentes del rendimiento el PMG mostró menor asociación con el rinde, mientras que los demás estuvieron muy estrechamente relacionados entre sí. La biomasa aérea lograda y el IC se mostraron fuertemente asociadas con el rendimiento en grano. Este comportamiento entre el PMG y el rendimiento concuerda con los resultados varios autores (Sakin et al., 2011; Mohammadi et al., 2011; González-Navarro et al., 2016). Sin embargo, Sakin et al., (2011) no encontraron una relación tan estrecha entre el $\mathrm{N}^{\circ}$ de granos por espiga y el $\mathrm{N}^{\circ}$ de espigas. $\mathrm{m}^{-2}$. En un trabajo realizado por Zarei et al., (2013) mostraron una asociación semejante a la encontrada aquí entre la biomasa aérea y el rendimiento en grano.

\section{Conclusiones}

a) La primera hipótesis se aceptó ya que hubo una reducción del rendimiento en granos del trigo candeal con la época de siembra tardía,

b) La segunda hipótesis se aceptó debido a que el tratamiento con agregado de fertilizante nitrogenado aumentó el rendimiento en grano en las dos fechas de siembra.

c) Se comprobó la existencia de variabilidad, recomendándose en la primera época a Buck zafiro y en segunda época a Buck esmeralda cuando existe una buena condición de fertilidad y con escasez de nitrógeno en la época tardía puede elegirse Bon. INTA Cariló. Buck esmeralda tiene estabilidad de rendimiento en grano en cualquiera de las dos épocas cuando las 
El efecto de la epoca de siembra, el genotipo y la fertilidad sobre el rendimiento en grano de trigo candeal en la región subhúmedaseca pampeana

condiciones de fertilidad son buenas.

\section{Bibliografía}

Abbate, P. E., Lázaro, L., \& Bariffi, H. (septiembre de 1998). Análisis comparativo de la determinación del número de granos entre trigo candeal y trigo pan. Actas XXII Reunión Argentina de Fisiología Vegetal. Mar del Plata, Argentina.

Anwar, J., Khan,S. B., Rasul, I., Zulkiffal, M., \& Hussain, M. (2007). Effect of sowing dates on yield and yield components in wheat using stability analysis. International Journal of Agriculture and Biology, 9 129-132.

Arduini, I., Pellegrino, E., \& Ercoli, L. (2018). Contribution of main culm and tillers to grain yield of durum wheat: Influence of sowing date and plant traits. Italian Journal of Agronomy, 13, 235-247.

Bell, M. A., \& Fischer, R. A. (1994). Guide to plant and crop sampling: measurements and observations for agronomic and physiological research in smal grain cereals. Wheat Special Report 32. CIMMYT, D. F., México.

Bozzini, A. (1988). Origin, distribution and production of durum wheat in the world. En G. Fabriani. \& C. Lintas (Eds.), Durum wheat: chemistry and technology. St. Paul, Min. EEUU: Am. Assoc. Cereal Chemist Inc.

Brancourt-Hulmel, M., Lecompte, C., \& Meynard, J. (1999). A diagnosis of yield-limiting factors on probe genotypes for characterizing environments in winter wheat trials. Crop Science, 39, 17981808.

Cantamutto, M. A., Agamennoni, R., Mockel, A. R., Vallatti, F. E., Matarazzo, R., \& Rivas, J. C. (1994) Comportamiento del trigo candeal en las condiciones ambientales del valle bonaerense de Rio Colorado. Revista de la Facultad de Agronomía UBA, 14, 101-106.

Dennett, M. D. (1999). Effects of sowing date and the determination of optimum sowing date. En E. H. Satorre \& G. A. Slafer (Eds.), Wheat ecology and physiology of yield determination. New York EEUU: Food Products Press.

Evans, L. T., \& Wardlaw, I. F. (1976). Aspects of the comparative physiology of grain yield in cereals. Advances in Agronomy, 28, 301-359.

Ferrante, A., Savin, R., \& Slafer, G. A. (2015) Relationship between fruiting efficiency and grain weight in durum wheat. Field Crops Research 177, 109-116.

Fischer, R. A. (1975). Yield potential in a dwarf spring wheat and the effect of shading. Crop Science, 15 607-613.

Fischer, R. A. (1985). Number of kernels in wheat crops and the influence of solar radiation and temperature. The Journal of Agricultural Science, 105, 447-461.

Forster, S. M., Ransom, J. K., Manthey, F. A., Rickertsen, J. R., \& Mehring, G. H. (2017). Planting date, seeding rate, and cultivar impact agronomic traits and semolina of durum wheat. American Journal of Plant Sciences, 8, 2040-2055.

González-Navarro, O. E., Griffiths, S., Molero, G. , Reynolds, P. M., \& Slafer, G. A. (2016). Variation in developmental patterns among elite wheat lines and relationships with yield, yield components and spike fertility. Field Crops Research, 196, 294-304.

González-Ribot, G., Opazo, M., Silva,P., \& Acevedo,E. (2017). Traits explaining durum wheat (Triticum turgidum L. spp. durum) yield in dry Chilean Mediterranean environments. Frontiers in Plant Science, 20(8), $1781 . \quad$ doi: 10.3389/fpls.2017.01781

Green, C. F., Paulson, G. A., \& Ivins, J. D. (1985). Time of sowing and the development of winter wheat. The Journal of Agricultural Science, 105, 217-221.

Larsen, A. O., \& Jensen, C. A. (2016). Análisis de rendimiento de trigo candeal en distintas épocas de siembra por ambiente y variedad. https://www.researchgate.net/publication/3033729 66

Loomis, R. S., \& Connor, D. J. (2002). Ecología de cultivos. Productividad y manejo en sistemas agrarios. Madrid, España: Ed. Mundi-Prensa.

MAGyP. (2018). Estimaciones Agrícolas. http:// datosestimaciones.magyp.gob.ar/reportes.php?re porte=Estimaciones. Visitada 1 diciembre de 2018.

Mohammadi, R., Armion, M., Sadeghzadeh, D., Amri, A \& Nachit, M. (2011). Analysis of genotype-byenvironment interaction for agronomic traits of durum wheat in Iran. Plant Production Science, 14, 15-21.

Otegui, M. E., \& López Pereira, M. (2003). Fecha de siembra. En E. H. Satorre, R. L. Benech Arnold, G. A. Slafer, E. B. de la Fuente, D. J. Miralles, M. E. Otegui \& R. Savin (Eds.), Producción de granos. Bases funcionales para su manejo. Buenos Aires, Argentina: Facultad de Agronomía de la UBA.

Pascale, A. J., \& Damario, E. A. (2004). Clasificación por tipos agroclimáticos del cultivo de trigo. En A. J. Pascale \& E. A. Damario (Eds.), Bioclimatología agrícola y agroclimatología. Buenos Aires, Argentina: Facultad de Agronomía Buenos Aires.

Patel, M. D, M.S. Dabhi, A.K. Patel, H.A. Desai \& C. Ram. 2018. Response of wheat varieties (Triticum aestivum L. and Triticum durum Desf.) to sowing time. International Journal of Current Microbiology and Applied Science, 7, 1555-1561.

Pei, X. X., Wang, J. A., Dang, J. Y., Wang, X. B., \& Zhang, D. Y. (2008). Effects of spikelet and grain position on fertile spikelet number, grain weight and quality of wheat. Scientia Agricultura Sinica, 41, 381-390.

Pimentel Gomes, F. (1978). Curso de estadística experimental. Buenos Aires, Argentina: Ed. Hemisferio Sur.

Tanaka, D. L., Krupinshy, J. M., Liepig, M. A., Merrill, S. D., Ries, R. E., Hendrickson, J. R., Johnson, H. A. 
Fernández, M. A., Gaggioli, C., y Repollo, R

\& Hanson, J. D. (2002). Dynamic cropping

systems: an adpatable approach to crop production in the Great Plains. Agronomy Journal, 94, 957-961.

Sakin, M. A., Akinci, C., Duzdemir, O., \& Donmez E. (2011). Assessment of genotype $x$ environment interaction on yield and yield components of durum wheat genotypes by multivariate analyses. African Journal of Biotechnology, 10, 2875-2885.

Soil Survey Staff. (2014). Claves para la Taxonomía de Suelos. Cap. 12: Molisoles. Dpto. Agric. de USAServ. Conservación Rec. Nat. (12nd ed.), USDA,

Spaner, D., Todd, A. G., \& McKenzie, D. B. (2000). The effect of seeding date and $\mathrm{N}$ fertilization on winter wheat yield and yield components in eastern New Foundland. Canadian Journal of Plant Science, 80,

Steel, R. G. D. \& Torrie, J. H. (1989). Bioestadística: principios y procedimientos. D.F. México: Ed. Mc Graw-Hill/ Interamericana.

Schwarte, A. J., Gibson, L. R., Karlen, D. L., Dixon, P. M., Liebman, M., \& Jannink, J. (2006). Planting date effects on winter triticale grain yield and yield components. Crop Science, 46, 1218-1224.

Zadoks, J. C., Chang, T. T., \& Konzak, C. F. (1974). A decimal code for the growth stages of cereals. Weed Research, 14, 415-421.

Zarei, L., Cheghamirza, K., \& Farshadfar, E. (2013). Evaluation of grain yield and some agronomic characters in durum wheat (Triticum turgidum L.) under rainfed conditions. Australian Journal of Crop Science, 7, 609-617. 